\title{
First Doppler images of the very young K2-dwarf PW Andromedae $=$ HD 1405 $5^{\star} \star \star$
}

\author{
K. G. Strassmeier ${ }^{1}$ and J. B. Rice ${ }^{2}$ \\ 1 Astrophysical Institute Potsdam, An der Sternwarte 16, 14482 Potsdam, Germany \\ e-mail: KStrassmeier@aip.de \\ 2 Department of Physics, Brandon University, Brandon, Manitoba R7A 6A9, Canada \\ e-mail: rice@BrandonU.ca
}

Received 21 March 2006 / Accepted 13 June 2006

ABSTRACT

\begin{abstract}
Aims. We present the first Doppler images of the single pre-main-sequence star PW And. Its position in the HR-diagram suggests it to be in the rapid-braking phase just prior to arrival on the ZAMS.

Methods. Images are obtained from seven individual spectral lines as well as from 5-nm wide chunks of spectrum that invert a total of 58 line profiles simultaneously.

Results. Cool spots with temperature differences with respect to the stellar photosphere of up to $1200 \mathrm{~K}$ are detected. Spot occurrence is mostly within an equatorial band up to $\pm 40^{\circ}$ of the stellar equator and thus contradicts magnetic-flux tube emergence models based on young K-star properties that predict an emerging latitude in two distinct bands of $+45-55^{\circ}$. This confirms previous suggestions that current magnetic-flux tube models predict emergence latitudes too low for G-dwarfs and too high for K-dwarfs, which may be caused by the fact that the G-dwarf models heavily rely on comparisons with the Sun. We also redetermine the absolute parameters of PW And in the light of a missing Hipparcos parallax and revise its age to be more near $20 \mathrm{Myr}$, in agreement with its logarithmic lithium abundance of $2.87 \pm 0.05$. A precise rotational period of $1.76159 \pm 0.00006$ was found from our photometric data in 2004 .
\end{abstract}

Key words. stars: activity - stars: starspots - stars: imaging - stars: individual: PW Andromedae - stars: pre-main sequence stars: fundamental parameters

\section{Introduction}

PW And is a rapidly-rotating single K2 dwarf within the Local Association moving group (Ambruster et al. 1994; Montes et al. 2003). Isochrone fitting of its position in the color-magnitude diagram suggests an age of just 30-80 Myr (López-Santiago et al. 2003 ) in agreement with its lithium abundance of nearly primordial value (3.0-3.4 on the $\log N(\mathrm{H})=12$ scale; Ambruster et al. 1994; Wichmann et al. 2003; López-Santiago et al. 2003). This suggests that PW And is likely still a pre-main-sequence star that had not had time enough to mix and burn its lithium, rather than being just a very young main-sequence star. Unfortunately, PW And lacks a Hipparcos parallax and is therefore difficult to place in the H-R diagram. Ambruster et al. (2003) made an attempt to compute absolute radii from the Barnes-Evans relation and the Stefan-Boltzmann law but obtained radii significantly smaller than the indirectly measured $R \sin i$ (from $v \sin i$ and the rotation period), which is clearly not physical and also suggestive of a pre-main-sequence nature.

The star is photospherically and chromospherically very active and already appeared in Bidelman's (1985) list of stars with Ca II H\&K and $\mathrm{H} \alpha$ emission. Hooten \& Hall (1990)

* Based on observations obtained at the Canada-France-Hawaii Telescope (CFHT) which is operated by the National Research Council of Canada, the Institut National des Sciences de l'Univers of the Centre National de la Recherche Scientifique of France, and the University of Hawaii.

$\star \star$ Figures A.1 to A.5 are only available in electronic form at http://www . aanda. org had determined a photometric rotation period of 1.745 days. Rotational line broadening was measured by several groups to be $21-23 \mathrm{~km} \mathrm{~s}^{-1}$ with various techniques. Just recently, a redetermination of absolute stellar parameters as well as extensive spectroscopic observations of activity indicators were presented by López-Santiago et al. (2003), and we refer the reader to this paper for further references. No direct detections of its surface magnetic field exist to date.

In this paper, we present the first Doppler image of PW And. Doppler imaging is a technique to reconstruct the surface temperature distribution from periodic variations of a star's spectral line profiles (e.g. Rice 2002). Over the one and a half decades since Doppler imaging of cool stars began, approximately 70 active cool stars have had one or more images of their surfaces (a recent listing is given by Strassmeier 2002). These stars include T Tauri, FK Comae, RS CVn-type stars as well as rapidlyrotating main-sequence stars. There are now comparisons that can be made among all the images of these stars, and in some cases there is even time tracking of images that allow us to see the time development of the active regions and in particular to note contrasts in behavior with the Sun.

\section{Observations}

\subsection{CFHT spectroscopy in 2004}

High-resolution spectroscopic observations were obtained with the f/8 Gecko spectrograph at the 3.6-m Canada-France-Hawaii $(\mathrm{CFH})$ telescope on Mauna Kea, Hawaii. Data were obtained over four nights (Aug. 26-27, 30-31), a gap of two nights was 
(a) Periodogram V+y

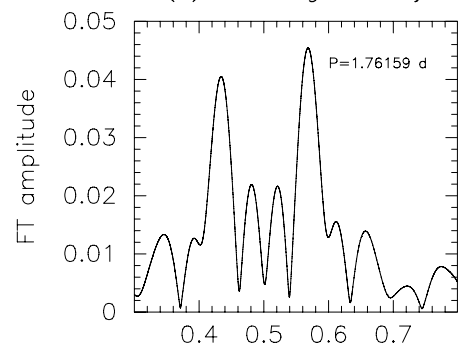

(b) $V+y$ photometry

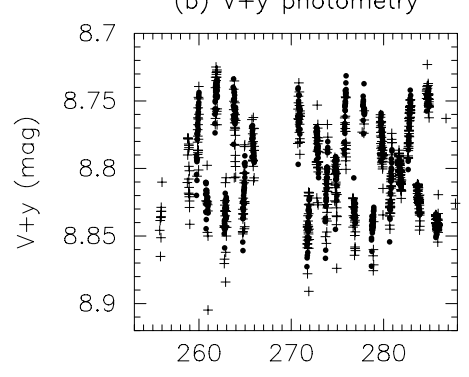

(c) Phased light curves

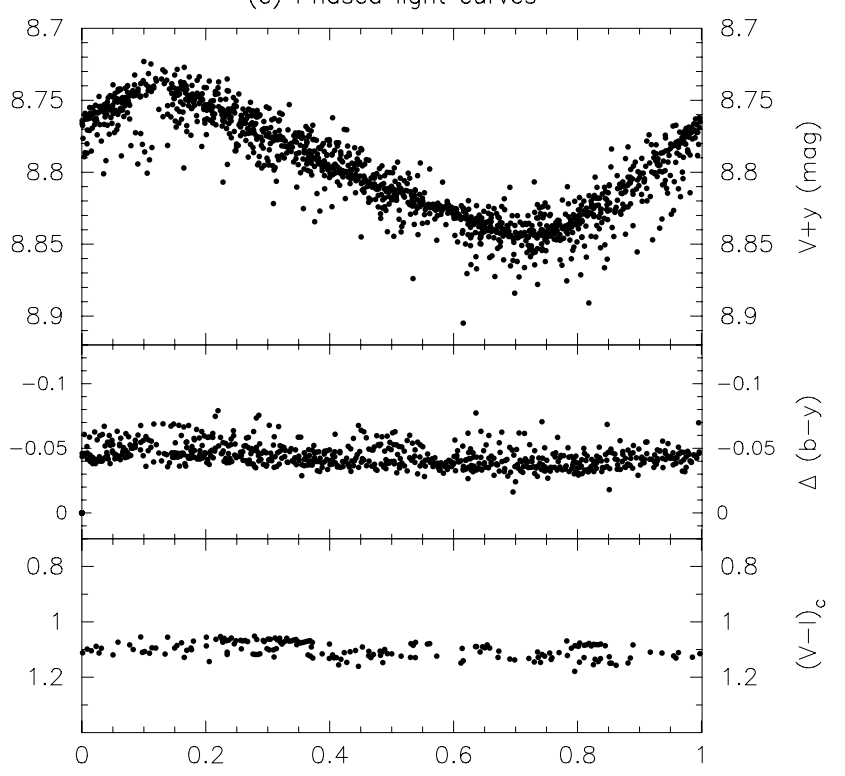

Fig. 1. New high-precision photometry of PW And in 2004. a) Periodogram from the combined $V$ and $y$ data. The $x$-axis is in units of cycles per day. b) $V$ (pluses) and $y$ (dots) photometry. The $x$-axis is in fractional days from JD 2453000 . c) Phased light and color curves with the ephemeris in Eq. (1). Note that the $b-y$ curve is differential with respect to HD 1440. The spectroscopic observations were obtained during the interval $2453243-50$. necessary to obtain optimum phase coverage. Now equipped with the CAFE fiber feed module (Baudrand \& Vitry 2000) Gecko provides a spectral resolution of 120000 in 8th order at $643 \mathrm{~nm}$ with a $5 \%$ better throughput in the red than with the mirror train. The spectrograph is not cross dispersed but, in combination with the $4600 \times 204813.5 \mu$ m-pixel EEV1 CCD, gives a $10 \mathrm{~nm}$ wavelength range. CAFE feeds light through a $27 \mathrm{~m}$-long $100 \mu \mathrm{m}$ Ceram-Optec fiber to a four-slice Bowen-Walraven image slicer before it enters the collimator. A single lithium spectrum was obtained with an order-sorting filter in the 9th order.

Seeing at CFHT was always between an astounding $0.30^{\prime \prime}$ to $0.8^{\prime \prime}$ and all integrations on PW And were set to an exposure time of $5 \times 600 \mathrm{~s}$. This allowed for a total of 27 spectra centered at $643.0 \mathrm{~nm}$ and one spectrum centered at $671.0 \mathrm{~nm}$ with an average signal-to-noise $(S / N)$ ratio of around 170:1 per single exposure per pixel. The time series also included five flat-field exposures per every target integration, totalling to up to 30-40 fairly evenly distributed flats per night. Twenty bias readouts at the beginning and at the end of each night and up to five integrations of a Th-Ar comparison-lamp spectrum throughout the night ensured a proper calibration of our spectra.

Stellar comparison targets were also observed with the same set-up, among them the bright and relatively inactive stars AX Mic $=$ HD202560 (M0V), $\epsilon$ Eri (K2V) and HD 166620 $(\mathrm{K} 2 \mathrm{~V})$ as well as the radial-velocity standard stars $\gamma$ Ser (F6V; $+6.58 \mathrm{~km} \mathrm{~s}^{-1}$ ) and $\alpha$ Ari (K2III; $-14.51 \mathrm{~km} \mathrm{~s}^{-1}$ ) (Scarfe et al. 1990).

All spectra were reduced and extracted using the Interactive Reduction and Analysis Facility (IRAF) provided by NOAO. More details of the standard reduction procedure were given in previous papers (e.g. Strassmeier \& Rice 2003).

\subsection{APT photometry}

High-precision photometry of PW And was obtained with the University of Vienna $0.75 \mathrm{~m}$ twin automatic photoelectric telescopes (APTs) at Fairborn Observatory in southern Arizona (Strassmeier et al. 1997). The T7-APT observed from JD 2452187 through 2453310 an achieved an external precision of 3.2 mmag, the T6-APT during the period 2453 259-286 achieved an external precision of 1.7 mmag. This included a dedicated one-month run shortly after the CFHT observations with both telescopes (from 2453 259-286). The T7-APT used Johnson $V I_{\mathrm{C}}$ filters, the T6-APT used Strömgren by filters. A total of $649 \mathrm{~V}, 455 I, 726 \mathrm{~b}$, and $736 y$ data points were obtained. The duty cycle for one group execution was $7.8 \mathrm{~min}$ and $9.8 \mathrm{~min}$ for T7 and T6, respectively. Integration time was $25 \mathrm{~s}$ in $V I$ and $30 \mathrm{~s}$ in by. All the measurements were done differentially with respect to HD $1440(B-V=1.06$; Hog et al. 2000). Check star was HD 1281 (K5). Johnson data are transformed to absolute values with the continuous observations of up 60 nightly standards. Strömgren data are just differentially. For further details on the observing procedure and the data reduction we refer to Granzer et al. (2001).

\section{Reexamining the astrophysical properties of PW And}

The only time-series photometry in the literature is that of Hooten \& Hall (1990) from photometric data taken during three observing seasons in 1985-88. Periods of $1.64 \pm 0.01,29 \pm 1$, and $1.745 \pm 0.005$ days were obtained for the three observing seasons, respectively. The authors recommended the 1.745-day period to be their most reliable since it had the least scatter. This period was adopted by López-Santiago et al. (2003) for their bisector analysis. Abbott et al. (1995) had obtained Four-College APT data in fall 1994 and quoted a photometric period of 1.75 days. However, from their abstract it is not clear whether this period was determined in that paper or just quoted.

Figure 1a shows the result from our Fourier analysis. The light curve is nearly sinusoidal and a FFT analysis seems appropriate. We applied the program package MUFRAN (Kolláth 1990), which is a collection of methods for analyzing multi periodic and unevenly sampled data. Standard discrete Fourier transforms of the light-curves were calculated, followed by a nonlinear least-square fit of the frequencies found. The combined $V$ and $y$ data were used and a period of $1.76159 \pm 0.00006$ days resulted in the lowest residuals. This period differs by just $0.9 \%$ from the Hooten \& Hall period but is almost a factor 100 more precise and thus supersedes this and all other previous values. 
We phase all data in this paper according to the following ephemeris

$\mathrm{HJD}=2453200.00+1.76159 \times E$,

where the zero point is an arbitrary time.

The rotational velocity $v \sin i$ of $\mathrm{PW}$ And was measured by several authors using differing techniques. Fekel (1997) obtained $23.4 \pm 10.0 \mathrm{~km} \mathrm{~s}^{-1}$ from broadening measurements of a number of unblended lines and an assumption of an appropriate radial-tangential macroturbulence. Griffin (1992) listed $21.5 \pm 1.0(\mathrm{rms}) \mathrm{km} \mathrm{s}^{-1}$ from Coravel traces. López-Santiago et al. (2003) determined $22.6 \pm 0.4 \mathrm{~km} \mathrm{~s}^{-1}$ from the width of the cross-correlation function of lines in an echelle spectrum versus an inactive reference star.

We have redetermined $v \sin i$ implicitly during the Doppler-imaging procedure. This takes into account the line-profile deformations due to spots, incorporates all lines in our spectra, and fits all rotational phases simultaneously, not just a single spectrum. Compared to other determinations this should, in principle, give the most accurate value. Our best value is $23.9 \pm 0.2 \mathrm{~km} \mathrm{~s}^{-1}$, where the error has been estimated from trial inversions and represents the sum of errors from, e.g. different abundances, continuum misplacement, imperfect wavelength and flat-field calibration, stray light in the spectrograph etc.

Together with the photometric period the minimum radius becomes $R \sin i=0.835 \pm 0.009 R_{\odot}$. Adopting our most-likely inclination of $i=46 \pm 6^{\circ}$ from the Doppler-imaging procedure (Sect. 4.2), the radius of PW And is $1.16_{-0.11}^{+0.15} R_{\odot}$. Note that inclinations between $40^{\circ}$ and $52^{\circ}$ are equally likely though. For comparison, a normal $\mathrm{K} 0-\mathrm{K} 2$ main sequence star is expected to have $0.81-0.75 R_{\odot}$ (e.g. Gray 1992); the ZAMS radii would be even slightly smaller. PW And is thus not a main-sequence star but rather a pre-main-sequence object, as suggested by López-Santiago et al. (2003). Using both the Stefan-Boltzmann law and the Barnes-Evans relation, Ambruster et al. (2003) had obtained radii smaller than the observed $R \sin i$ which, of course, as they mentioned themselves, is unphysical. In the light of our more precise values, the problem must lie with the assumption of the unspotted brightness and color that must be adopted for the two methods.

The brightness-averaged surface temperature from our maps in Sect. 4.2 is $4800 \mathrm{~K}$. Due to the lack of a Hipparcos parallax, we must use this temperature and above absolute radius to obtain the luminosity of $0.640 L_{\odot}$. This is likely a lower value because it is based on the brightness-averaged temperature of our single Doppler image in 2004.7. If the star were seen without its spots its effective temperature may be $\approx 5000 \mathrm{~K}$, and its luminosity correspondingly $0.753 L_{\odot}$. The difference suggests a missing energy of $4 \times 10^{32} \mathrm{erg} \mathrm{s}^{-1}$, which would make the star $8 \%$ more massive than quoted below. A comparison with the evolutionary tracks and isochrones from Baraffe et al. (1998) suggests a mass of $1.07 M_{\odot}$ and an age of $\approx 20 \mathrm{Myr}$. As a comparison, the D'Antona \& Mazzitelli (1997) tracks favor more a mass of $1.10 M_{\odot}$.

We have also measured the Li I 670.8-nm feature in a single $R=120000, S / N \approx 200$ spectrum (Fig. 2) and obtained an equivalent width of $278 \pm 4 \mathrm{~m} \AA$ after subtracting a spectrum of the K2-dwarf $\epsilon$ Eri. The latter contributes $22 \mathrm{~m} \AA$ over the total width of the lithium line in PW And, as does a similar spectrum of the K0-dwarf 70 OphA. The non-LTE curves of

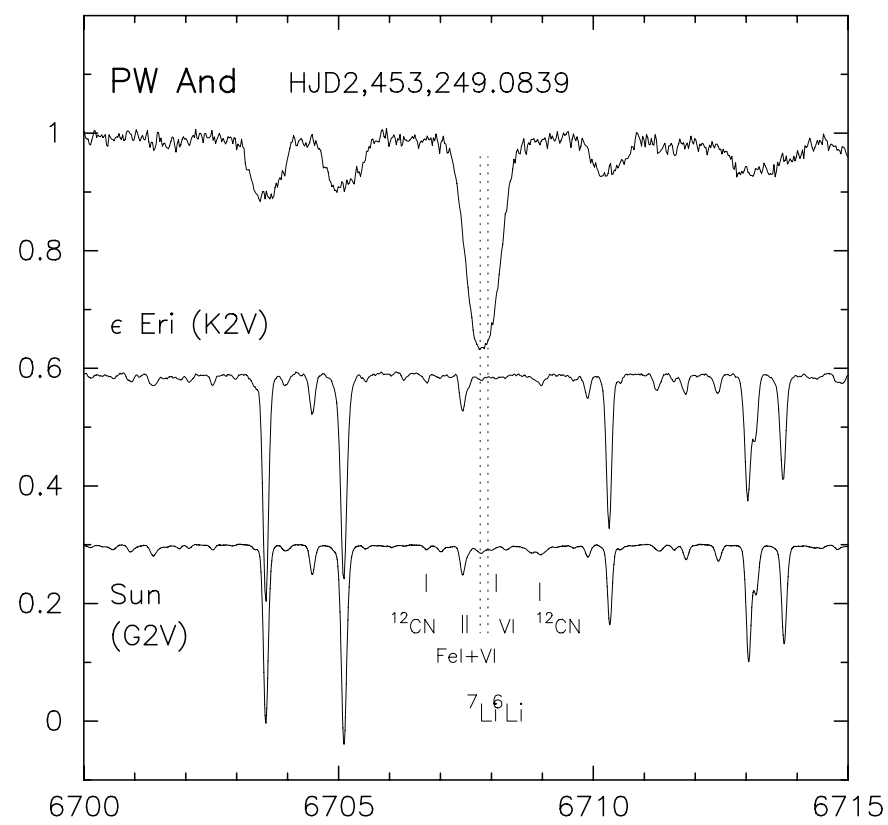

Fig. 2. A lithium spectrum of PW And at $R=120000$ (top). The $\mathrm{Li}$ I $670.8 \mathrm{~nm}$ line is the strong line in the center. Its equivalent width is $278 \mathrm{~m} \AA$ corrected for the contribution from an inactive star $\epsilon$ Eri (middle spectrum) or 70 Oph A (not shown). As a comparison, a $R=600000$ spectrum of the Sun is shown at the bottom (Kurucz et al. 1984). Notice the line-core asymmetries in PW And. All spectra are on the same intensity scale but $\epsilon$ Eri and the Sun are shifted for plotting purposes.

growth of Pavlenko \& Magazzú (1996) for a $5000 \mathrm{~K} / \log g=$ 4.5 model convert this equivalent width into a logarithmic $\mathrm{Li}$ abundance of $2.87 \pm 0.05$ (on the $\log n(\mathrm{H})=12.00$ scale). In case we use a $4750 \mathrm{~K} / \log g=4.5$ model, which is more like the brightness-averaged surface temperature of PW And, the conversion gives 2.60 dex. These abundances are significantly smaller than the previous determination of 3.4 dex by LópezSantiago et al. (2003) based on $T_{\text {eff }}=4900 \mathrm{~K}$, but likely still in agreement with the 3.0 dex given by Ambruster et al. (1994). Our corrected equivalent width is also in very good agreement with the value of $273 \mathrm{~m} \AA$ listed by Wichmann et al. (2003) and $267 \mathrm{~m} \AA$ listed by López-Santiago et al. (2003). The discrepancy thus likely comes from the curve-of-growth calibration adopted by López-Santiago et al. and the inherent uncertainties of the Pavlenko \& Magazzú (1996) NLTE calibration.

\section{Doppler imaging of PW And}

\subsection{The TempMap code and its assumptions}

TempMap computes an error function that represents the degree to which the predicted spectrum from a current trial image of a star (starting with a blank surface) differs from the observed spectrum and then altering the parameters of the image to iteratively reduce the error function to a minimum consistent with the error of the observations. The squared difference between the forward calculation and the observation is summed over all wavelengths and a full set of observations spaced in time through the full cycle of phases of the star's rotation. A regularizing functional of the minimization process - maximum entropy in our case - is used so that the line profiles calculated from the image produced do not overfit the observed spectra. For a full discussion of Doppler Imaging and the process of regularization see 
Table 1. Summary of astrophysical data.

\begin{tabular}{|c|c|c|}
\hline Parameter & PW And & Reference \\
\hline Spectral type & $\mathrm{K} 2 \mathrm{~V}$ & López-Santiago et al. \\
\hline age & $\begin{array}{l}\text { 30-80 Myr } \\
\approx 20 \mathrm{Myr}\end{array}$ & $\begin{array}{l}\text { López-Santiago et al. } \\
\text { this paper }\end{array}$ \\
\hline$b-y$ & 0.535 & Nordström et al. (2004) \\
\hline$(V-I)_{C}$ & 1.06 & this paper \\
\hline $\log g$ & 4.5 & (adopted) \\
\hline$T_{\text {phot }}$ & $5000 \mathrm{~K}$ & this paper \\
\hline$T_{\text {average }}^{1}$ & $4800 \pm 50 \mathrm{~K}$ & this paper \\
\hline$v \sin i$ & $23.9 \pm 0.2 \mathrm{~km} \mathrm{~s}^{-1}$ & this paper \\
\hline Rotation period & $1.76159 \pm 0.00006$ & this paper \\
\hline Inclination & $46^{\circ} \pm 6^{\circ}$ & this paper \\
\hline Radius & $1.16_{-0.11}^{+0.15} R_{\odot}$ & this paper \\
\hline Luminosity & $0.64 L_{\odot}$ & this paper \\
\hline Mass & $1.07 M_{\odot}$ & this paper \\
\hline $\log n(\mathrm{Li})$ & $2.87 \pm 0.05$ & this paper \\
\hline $\log n(\mathrm{Fe})$ & $7.50 \pm 0.02$ & this paper \\
\hline $\log n(\mathrm{Ca})$ & $6.02 \pm 0.02$ & this paper \\
\hline
\end{tabular}

${ }^{1}$ Brightness-averaged temperature from the Doppler images.

Rice (2002) and Piskunov \& Rice (1993) and for TempMap tests see Rice \& Strassmeier (2000).

In the current application, the local line profiles were calculated from ten Kurucz (1993) model atmospheres covering $3500 \mathrm{~K}$ to $5500 \mathrm{~K}$ of fixed $\log g$ of 4.5 for the purpose of recovering the local effective temperatures. Photometry was not included in the inversions because our APT data were not strictly simultaneous with the spectroscopy. A more detailed account of the numerical procedure and a recent application to a cool premain-sequence star can be found in Strassmeier et al. (2005).

Microturbulence was determined from Blackwell-diagrams as prescribed by Gray (2000). The equivalent widths of ten iron lines were measured and converted to an iron abundance as a function of microturbulence with a curve-of-growth analysis. These abundances show that all lines seem to converge at a microturbulence of $2 \mathrm{~km} \mathrm{~s}^{-1}$ and an optimal value for the abundance of iron of 7.5 (on the usual $\log n(\mathrm{H})=12.00$ scale). We note that this is in discordance with the metallicity of -0.78 dex listed in the Geneva-Copenhagen catalog (Nordström et al. 2004).

Table 1 again summarizes the relevant astrophysical data of PW And.

\subsection{Single-line inversion and full-spectrum inversion}

We first proceeded with a line-by-line inversion and its parameter optimization. Our major lines were Ca I 643.9 (363), Fe I 643.0 (244), Fe I 642.1 (228), Fe I 641.9 (166), Fe I 641.1 (267), Fe I 640.8 (218), Fe I+Fe I 640.0 (417), and Fe I 639.3 (250), where the number in parenthesis is the average equivalent width in $m \AA$, errors are usually $\pm 3-4 \mathrm{~m} \AA$. Plots of the full wavelength range of similar stars were shown in previous papers, e.g. in Strassmeier \& Rice (2003). We abandoned the close iron blend at $640.0 \mathrm{~nm}$ from our input because of resulting uncertainties of its central wavelength and relative line strength due to the likely contamination from a weak $\mathrm{CN}$ line. Note that the other two close iron lines at $641.94 \mathrm{~nm}$ (excitation potential of $4.7 \mathrm{eV}$ ) and $642.13 \mathrm{~nm}$ (excitation potential of $2.2 \mathrm{eV}$ ) are solved for simultaneously from the beginning and show consistent results.

The fitting errors that we achieve for the individual lines and line pairs are comparable, e.g. $8.2 \times 10^{-3}$ for the
Fe I $641.9+642.1$ pair corresponding to a $S / N$ ratio of $122: 1$. The ideal fit would be achieved for $S / N$ ratio that matches the observed one, i.e. on average 170:1. Error is defined here as $\sqrt{\sum(\mathrm{O}-\mathrm{C})^{2} /(n-1)}$, where $n$ is the number of spectrum points in all of the observations, i.e. the number of pixels per phase times the number of phases. This number of data points is such that the problem is essentially over-determined and the leastsquares fit predominates. We have two to three times as many profile points per star in total as we have pixels (2592) visible on the surface of each star. Although the strong Ca I 643.9 line could be fitted to the same level, its surface map remained markedly different: besides the correct recovery of the main features seen in the other lines, a circumpolar ring warmer by $\approx 400 \mathrm{~K}$ than $T_{\text {eff }}$ and a tiny, 10-degree diameter, cool polar spot remained in the $\mathrm{Ca}$ image. We are confident that these are partially imaging artifacts coming from, i) the high sensitivity of the local-line strength of Ca I 643.9 at the temperature of PW And; ii) the small rotational broadening compared to the large intrinsic width of the line and; iii) is caused by a chromospheric contribution to the line profile. The latter makes also Fe I 643.0 an error-prone line, although its recovery is not as strikingly different than from Ca. The Ca I 643.9-nm local line width is $40 \%$ higher (FWHM $0.145 \AA$ ) than for the other Fe I lines, except Fe I 643.0, which is $20 \%$ wider. Therefore, we assigned half weight for both lines for the average map and for the full-spectrum inversion.

We run a grid of solutions for each line for $v \sin i$ and inclination $i$ with fixed microturbulence, abundances, and transition probabilities. Overall best fits were achieved with $v \sin i$ of $23.86 \pm 0.22 \mathrm{~km} \mathrm{~s}^{-1}$ and $i=46.0^{\circ} \pm 6.5^{\circ}$. Errors are rms values from the different lines and line pairs and just suggest the ranges within the true value must lie.

Our final averaged image is shown in Fig. 3. It is a brightness averaged temperature map from Ca I 643.9 (half weight), Fe I 643.0 (half weight), Fe I 642.1, Fe I 641.9, Fe I 641.1, Fe I 640.8, and Fe I 639.3. Representative data and the fits for one line pair - Fe I $642.1 \mathrm{~nm}$ and Fe I $641.9 \mathrm{~nm}$ - are shown in Fig. 5. It appears there are unknown weak lines causing a depression between these two iron lines with respect to the fit shown. We could not identify these blending lines assuming solar abundances. Therefore, this region was given zero weight during the inversion. Respective fits for all wavelength regions are given in the electronic appendix (Figs. A.1-A.5). The brightness-averaged surface temperature from all spectral lines is $4800 \pm 50 \mathrm{~K}$ (rms) with a range of surface temperatures between $3780 \mathrm{~K}$ and $5200 \mathrm{~K}$. The photospheric temperature is of course higher than the brightness-averaged value and we estimate it more like $5000 \mathrm{~K}$. Only few surface pixels were recovered with $5200 \mathrm{~K}$, and we discharge them as artificial.

We then proceed with the inversion of the entire wavelength range of our spectra $(639.2-644.0 \mathrm{~nm})$. Each rotational phase then consists of a data vector of length 3060 wavelength points or $5.5 \mathrm{~nm}$. It contains the major imaging lines listed above plus a total of 51 blends. A weight list is added to TEMPMAP in which each wavelength pixel is assigned a weight. Per default the file consists of 3060 unit weights, but the regions outside of $\pm(2 v \sin i+0.1 \mathrm{~nm})$ of a line center, i.e. all mostly continuum, were given zero weight. The CaI 643.9-nm and the Fe I 643.0-nm lines were given half weight again, see above. The rest remained at unity. Our final image with the full-spectrum synthesis approach is shown in Fig. 4. Because the fits for a particular line are not easily distinguishable by eye from the singleline fits, we do not give a separate figure. 

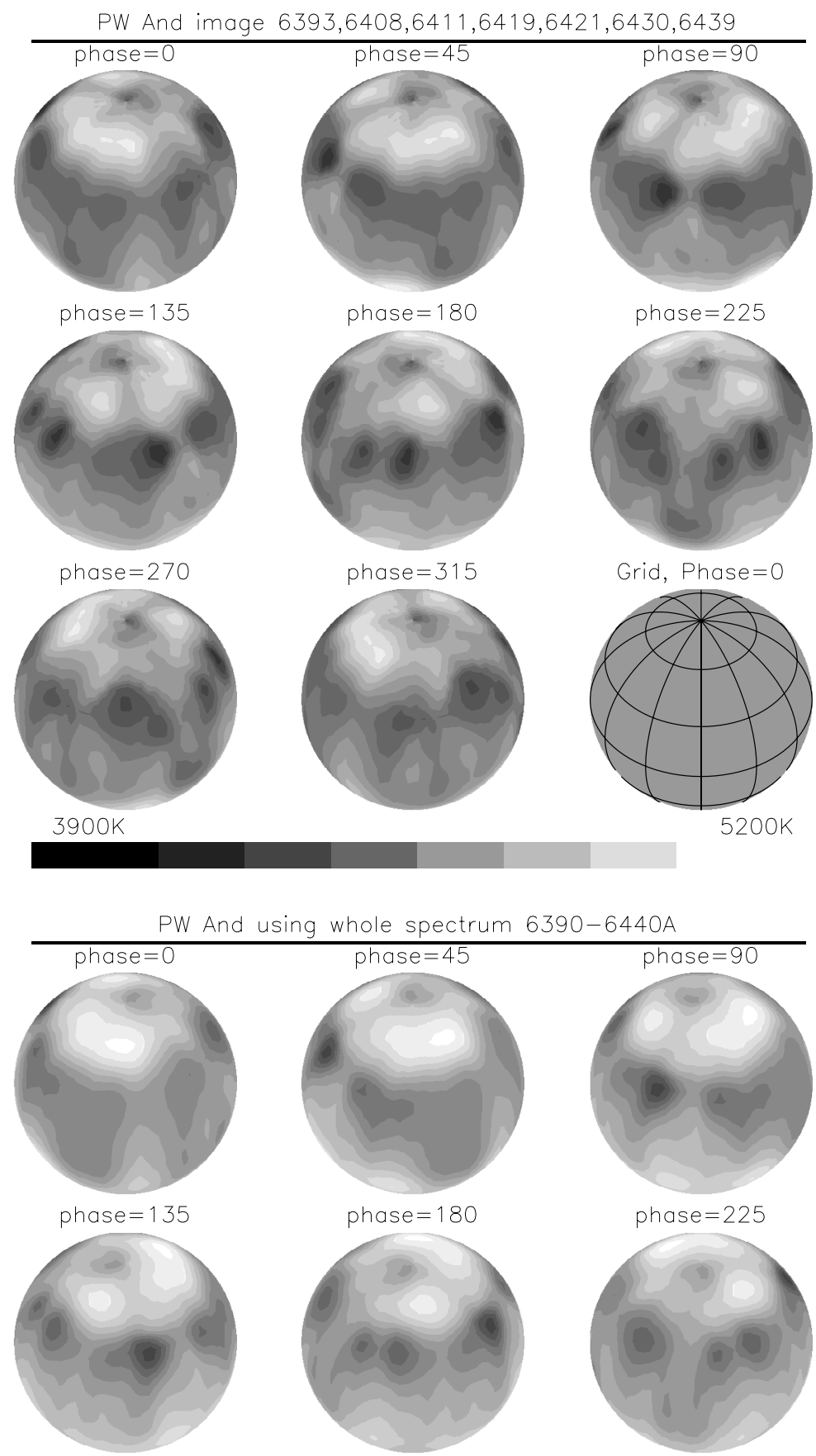

phase $=270$

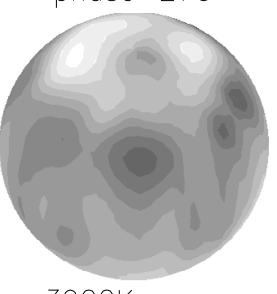

$3900 K$

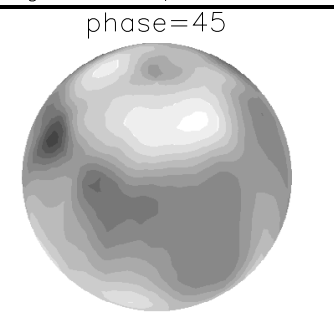

phase $=180$

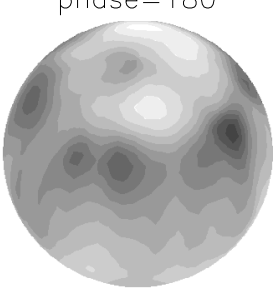

phase $=315$

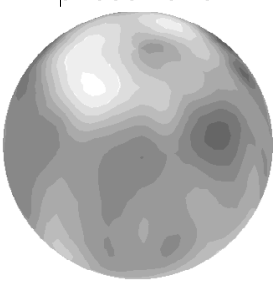

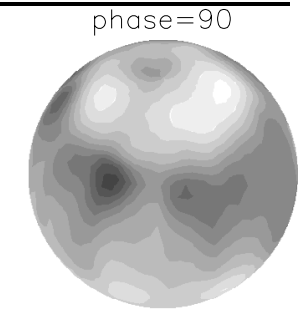

phase $=225$

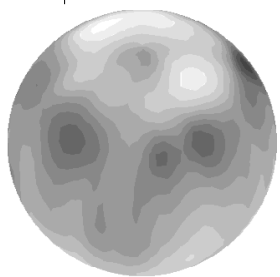

Grid, Phase $=0$

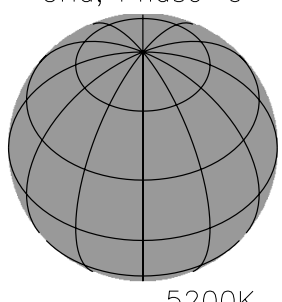

Fig. 3. Averaged Doppler image of PW And for August 26-31, 2004 (JD 2453 243-249). Maps are plotted in a spherical projection at eight equidistant rotational phases. The temperature scale is indicated and is the same for all projections. The image is an average from seven spectral regions, see text. Note that the photospheric temperature is $\approx 5000 \mathrm{~K}$.

Fig. 4. Doppler image of PW And from the fullspectrum approach. Otherwise as in Fig. 3.

\subsection{Results}

PW And does not show a cool polar spot nor any distinctive high-latitude spots. The maps are dominated by several lowlatitude spots between latitudes of $+40^{\circ}$ and $-20^{\circ}$ (the latter close to the limit of visibility at $-44^{\circ}$ ) and temperatures of $\approx 1200 \mathrm{~K}$ below the photospheric value. The locations of these spots are consistently recovered in all single-line inversions as well as in the full-spectrum inversion. We are therefore quite confident about their reality. Its recovered contrasts vary by approximately $\pm 100 \mathrm{~K}$ from line to line but agree to within $\pm 20 \mathrm{~K}$ in the average map and the full-spectrum map. 

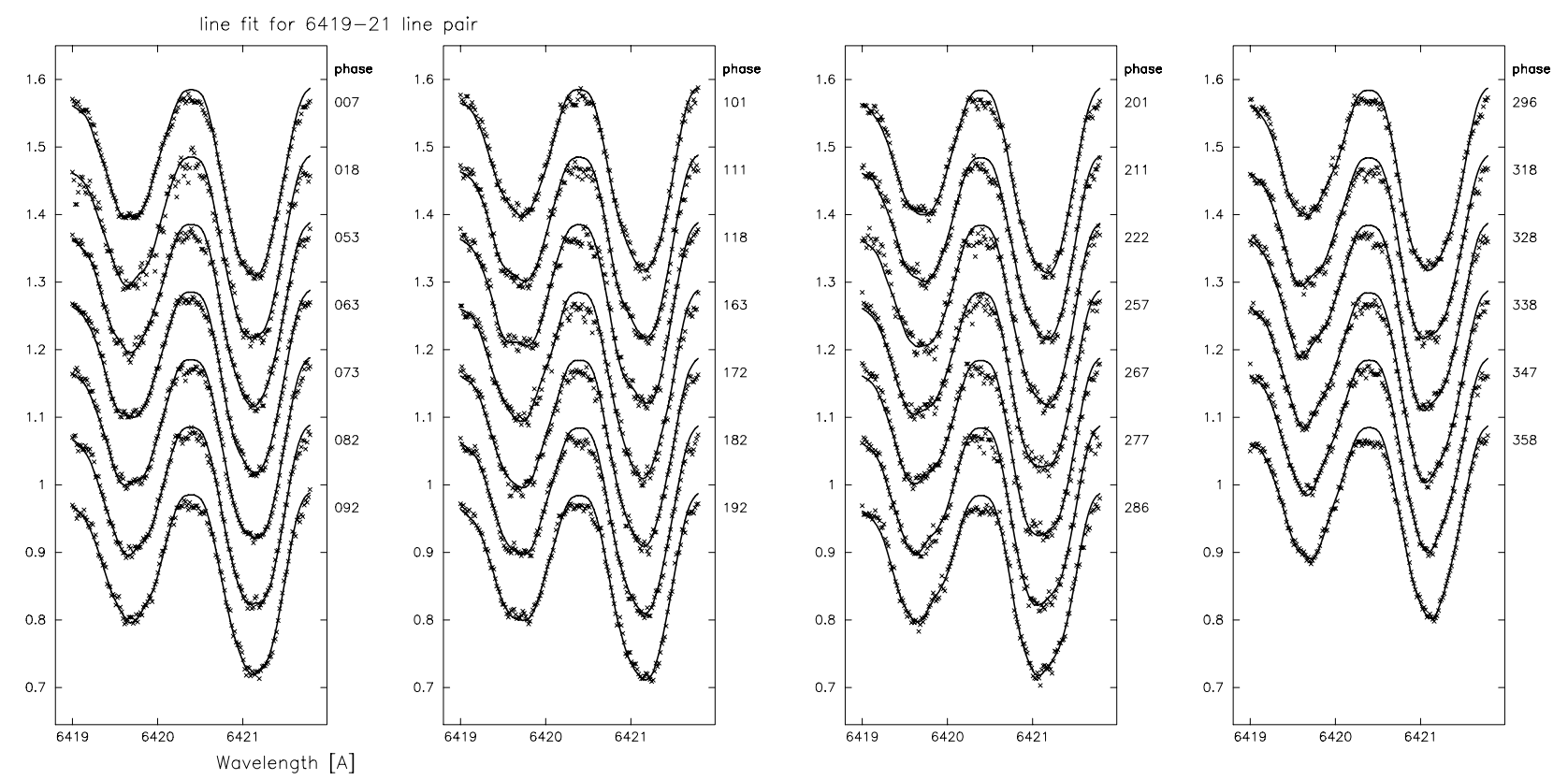

Fig. 5. Example line-profile fits. Shown is only the (simultaneous) inversion of the Fe I 642.1 and Fe I 641.9 line pair for all 27 phases. Phase is indicated in degrees $\left(0-360^{\circ}\right)$ on the right side of the individual graphs. Crosses are the data, the lines are the fits. The points between the two lines where the fit is poor were given zero weight in order not to influence the solution.

The visible polar region shows two unusual warm features with average relative temperatures of up to $200 \mathrm{~K}$ above the photospheric temperature (at longitudes of roughly $20^{\circ}$ and $150^{\circ}$ and a latitude of $+60^{\circ}$; see Figs. 3 and 4 ). These regions appear to be significant because they show up to some extent in the recovery from all lines. The high contrast in the average and the full-spectrum map is mostly due to the inclusion of the Ca I 643.9-nm line though and, although to a lesser extent, also the Fe I 643.0-nm line, despite that we gave them only half weight. A recovery with zero weight and full weight did not change the resulting contrast significantly. The other lines recover these features with a temperature difference of no more than 150-200 K above the photospheric temperature and, usually, would not be considered significant.

Figure 6 compares latitudinally averaged temperatures from the single-line maps. It demonstrates that the main spot activity on PW And takes place below latitudes of $+40^{\circ}$. There is some evidence of spot activity below the equator but the Dopplerimaging technique is not capable of recovering accurate latitudes close to the visibility limit. Therefore, we can not conclude that there exists indeed a symmetric spot-activity belt as on the Sun.

\section{Discussion and conclusions}

The overall spot morphology of PW And resembles that of today's Sun. Activity seems to be concentrated below $+40^{\circ}$ latitude and consists of at least six individual cool spots. Spot sizes are 10-100 times larger than solar spot groups, despite a solarlike radius of $1.16 R_{\odot}$. Two regions $\approx 200-\mathrm{K}$ warmer than the photosphere at latitudes of approximately $+60^{\circ}$ could be interpreted as high-latitude chromospheric plages that are still seen in the stronger photospheric lines. Although PW And is a very young, likely pre-main-sequence $\operatorname{star}(\approx 20 \mathrm{Myr})$, we see no evidence of mass accretion in its optical spectrum. The alternative interpretation of warm spots due to accretion impacts from a circum-stellar disc onto the stellar surface seems therefore not

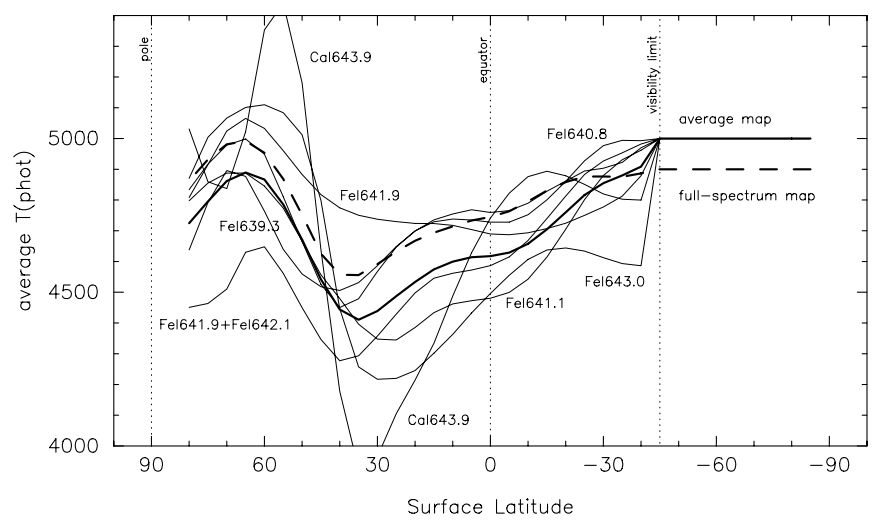

Fig. 6. Latitudinally averaged surface temperatures. Shown is the average temperature per 5-degree latitude bin between the surface visibility limit at $-44^{\circ}$ and close to the visible pole for the individual spectral lines images (the thick line is the average from the combined image). The surface pixels around the geometric pole between $85-90^{\circ}$ are not plotted. The main spot activity seems to occur at a latitude of below $\approx+40^{\circ}$ while the warm features seem to reside at a latitude of $\approx+60^{\circ}$, especially well seen in the Ca I 643.9-nm line. appropriate (but see Strassmeier et al. 2005 for a classical T Tauri star).

One of the theoretically interesting comparisons we can make is to see if the model predictions for the latitudinal location of the active regions (Schüssler et al. 1996) fit well with observations. Generally, the work of Schüssler et al. and others involves calculating the trajectory of the magnetic flux tubes throughout the convective envelope from their source within the overshoot layer between the radiative core and the envelope up to the surface where they become visible. Granzer et al. (2000) expanded this work to pre-main-sequence stars of masses between 0.4 to $2.4 M_{\odot}$. For stars of comparable rotation rate, the stars with deeper convection envelopes should have their surface spot activity predominantly at higher latitudes. 

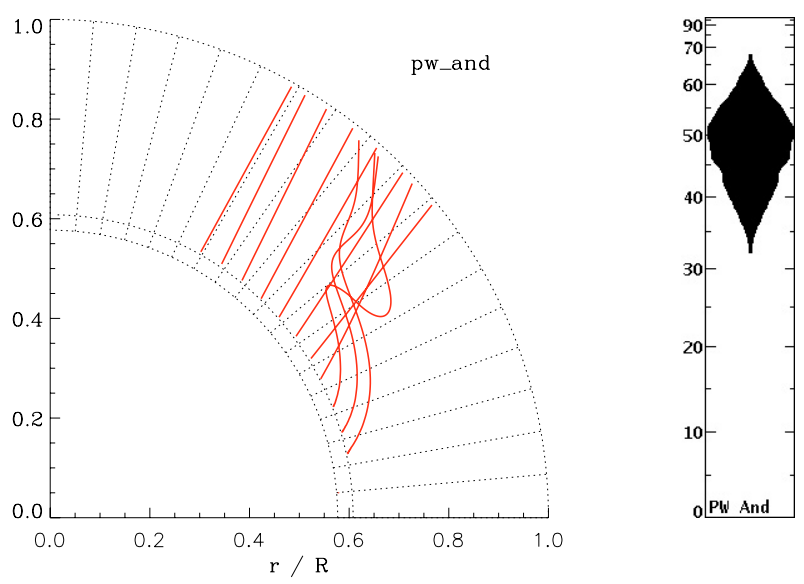

Fig. 7. The expected latitude distribution of emerging flux tubes for PW And. Shown is as a radial cross section with the trajectories of the summit of rising flux loops (lines; left panel). Its surfacing probability as a function of latitude between zero and $90^{\circ}$ is shown in the right panel (a larger width means a higher surfacing probability). Most magnetic flux would be expected to surface at a latitude of $+45-55^{\circ}$.

Figure 7 shows the calculation results for a model based on the PW And parameters in Table 1. Its convection zone reaches $60 \%$ of the stellar radius and is assumed to behave as a rigid rotator. The initial field strength at the bottom of the convection zone is chosen so that the magnetic flux reaches values typical for large solar active regions $\left(10^{14} \mathrm{~Wb}\right)$. For more details we refer to Granzer et al. (2000). The model predicts the majority of the magnetic flux to surface at a latitude of $50 \pm 10^{\circ}$, in obvious disagreement with the Doppler observations that show most spots spread between $0-40^{\circ}$ with a tendency towards $+30^{\circ}$. Most convincingly, the model does not predict any flux below a latitude of $\approx 30-35^{\circ}$, in disagreement with our observation. Some flux tubes are predicted to emerge even at latitudes as high as $+70^{\circ}$. The question whether it is the "warm" features, i.e. the ones near a latitude of $+60^{\circ}$ in our image, that are to be compared to the flux-tube models rather than the cool spots remains to be determined. After all, solar analogy may not apply and the warmer plage-like features could carry most of the magnetic flux.

In several of our earlier papers (e.g. Rice \& Strassmeier 1998 and Kövári et al. 2004), we showed that the activity on two young main-sequence stars, LQ Hya (K2V) and EK Dra $(\mathrm{G} 1.5 \mathrm{~V})$, seemed to run counter to this expectation. This was confirmed for the very young G0-star HD171488 (Strassmeier et al. 2003) along with a number of other G-dwarfs from the literature (see Table 4 in above paper). For a star as cool as LQ Hya (K2), one would have expected the deeper convection envelope and thus higher-latitude spot activity, yet the activity seemed more concentrated to the equator than for the early G dwarfs EK Dra and HD171488, where the activity was more in the form of a polar cap. On the contrary, the images in Donati et al. (2003) for 1998-2001 did show a small polar spot simultaneous with low latitude spots. PW And (K2) is a bit younger and more massive but otherwise comparable to LQ Hya, and shows predominantly equatorial active regions counter to the expectations from models.

Are PW And and LQ Hya directly comparable? PW And is more massive than LQ Hya, $1.07 M_{\odot}$ versus $0.8 M_{\odot}$, it is possibly also younger, $\approx 20-30 \mathrm{Myr}$ compared to $\approx 100 \mathrm{Myr}$ of LQ Hya, and marginally cooler, $T_{\text {eff }}$ of $5000 \mathrm{~K}$ compared to $5070 \mathrm{~K}$ of LQ Hya (each $\pm 50-100 \mathrm{~K}$ though). Its lithium abundances are also very similar $(\approx 2.8-2.9 \mathrm{dex})$ and the rotation periods are even within a few percent. We could therefore assume similar interior structure and predict the same preferential flux-tube surfacing latitudes than for LQ Hya (shown in Fig. 8 in Kôvári et al. 2004). These simulations predict a most probable surfacing latitude of $40^{\circ}$, with a total range of possible emergence latitudes of between $30^{\circ}$ and $70^{\circ}$. This is only in agreement with the observation that neither PW And nor LQ Hya shows a cool polar spot. The model still fails to predict truly equatorial spots though, as we mostly see on PW And and on LQ Hya. However, this may be a meaningless inconsistency because of the difficulty for the recovery to achieve very accurate latitudes, i.e. better than one resolution element on the stellar surface. Also, because the flux tube simulations start in the super-adiabatic layer beneath the convection zone, the surfacing latitudes could be questioned because of the questionable applicability of a pure interface-layer dynamo to very active latetype stars (see, e.g., Saar 1998). On the low-mass end of the ZAMS, Mullan \& MacDonald (2001) suggested that even a M6 star should still have a radiative core and therefore an interfacedynamo component. At the spectral type of $\mathrm{K} 2$ and the mass of $1.07 M_{\odot}$ of PW And, we would expect the interface component to dominate. Furthermore, as suggested by Mackay et al. (2004), the values for surface meridional flow must be increased by around a factor of $10\left(\approx 100 \mathrm{~m} \mathrm{~s}^{-1}\right)$ in order to produce magnetic flux at high latitudes, which then would make truly equatorial spots even less likely.

Once stellar models incorporate magneto-convection, stellar evolutionary tracks may become distinctively different. Mullan \& MacDonald (2001) found that for two stars with the same mass (valid for masses up to $0.6 M_{\odot}$ ), the magnetic star has a larger radius and a smaller effective temperature than the nonmagnetic star. So far, we can not quantify this for very young stars with masses above $0.6 M_{\odot}$. However, Ambruster et al. (2003) speculate that the active, Pleiades-age K dwarfs are actually more massive than their spectral class indicate. If so, our interior models used to host the flux tube emergence (cf. Granzer et al. 2000) may be inappropriate as well and, more importantly, the flux tube's starting points in the super-adiabatic layer would be wrong in the first place. We speculate that this could have a dramatic impact for the predicted surface latitudes of flux-tube emergence.

Acknowledgements. J.B.R. acknowledges support from the Natural Science and Engineering Research Council of Canada (NSERC). K.G.S. is grateful to the German Science Foundation (DFG) for support under grant STR645/1-1. We both thank Nadine Manset and the other CFHT staff for their continuous support. Thanks also to Dr. Thomas Granzer for computing the flux-tube models for us.

\section{References}

Abbott, B. P., Pomerance, B. H., \& Ambruster, C. W. 1995, BAAS, 27, 842 Ambruster, C. W., Brown, A., \& Fekel, F. C. 1994, in 8th Cool Stars, Stellar Systems, and the Sun, ed. J.-P. Caillault, PASPC 64, 348

Ambruster, C. W., Fekel, F. C., \& Brown, A. 2003, in 12th Cool Stars, Stellar Systems, and the Sun, ed. A. Brown, G. Harper, \& T. Ayres, Univ. of Colorado, 912

Baraffe, I., Chabrier, G., Allard, F., \& Hauschildt, P. H. 1998, A\&A, 337, 403

Baudrand, J., \& Vitry, R. 2000, Proc. SPIE, 4008, 182

Bidelman, W. P. 1985, AJ, 90, 341

D’Antona, F., \& Mazzitelli, I. 1997, in ed. R. Pallavicini, \& G. Micela, Cool stars in clusters and associations, Mem. S. A. It., 68, 807

Donati, J.-F., Collier Camerosn, A., Semel, M., et al. 2003, MNRAS, 345, 1145 Fekel, F. C. 1997, PASP, 109, 514

Granzer, T., Caligari, P., Schüssler, M., \& Strassmeier, K. G. 2000, A\&A, 355, 1087

Granzer, T., Reegen, P., \& Strassmeier, K. G. 2001, AN, 322, 325 
Gray, D. F. 1992, The observation and analysis of stellar photospheres, CUP, 431 Gray, R. O. 2000, http://www.phys .appstate.edu/spectrum Griffin, R. F. 1992, Observatory, 112, 41

Hog, E., Fabricius, C., Makarov, V. V., et al. 2000, A\&A, 355, L27

Hooten, J. T., \& Hall, D. S. 1990, ApJS, 74, 225

Kolláth, Z. 1990, The program package MUFRAN, Occasional Technical Notes of Konkoly Observatory, No. 1 (www. konkoly.hu/Mitteilungen/...\#TechNotes)

Kövári, Zs., Strassmeier, K. G., Granzer, T., et al. 2004, A\&A, 417, 1047

Kurucz, R. L. 1993, ATLAS-9, CD-ROM \#13

Kurucz, R. L., Furenlid, I., Brault, J., \& Testerman, L. 1984, NSO Atlas No. 1, Solar flux atlas from 296 to $1300 \mathrm{~nm}$

López-Santiago, J., Montes, D., Fernández-Figueroa, M. J., \& Ramsey, L. W. 2003, A\&A, 411, 489

Mackay, D. H., Jardine, M., Collier Cameron, A., Donati, J.-F., \& Hussain, G. A. J. 2004, MNRAS, 354, 737

Montes, D., López-Santiago, J., Fernández-Figueroa, M. J., \& Gálvez, M. C. 2003, A\&A, 379, 976

Mullan, D. J., \& MacDonald, J. 2001, ApJ, 559, 353
Nordström, B., Mayor, M., Andersen, J., et al. 2004, A\&A, 418, 989

Pavlenko, Ya. V., \& Magazzú, A. 1996, A\&A, 311, 961

Piskunov, N. E., \& Rice, J. B. 1993, PASP, 105, 1415

Rice, J. B. 2002, AN, 323, 220

Rice, J. B., \& Strassmeier, K. G. 1998, A\&A, 336, 972

Rice, J. B., \& Strassmeier, K. G. 2000, A\&AS, 147, 151

Saar, S. H. 1998, in ed. R. Donahue, \& J. Bookbinder, Cool Stars, Stellar Systems, and the Sun, ASPC 154, 211

Scarfe, C. D., Batten, A. H., \& Fletcher, J. M. 1990, Publ. Dominion Astron. Obs. Victoria, 18, 21

Schüssler, M., Caligari, P., Ferriz-Mas, A., Solanki, S., \& Stix, M. 1996, A\&A, 314,503

Strassmeier, K. G. 2002, AN, 323, 309

Strassmeier, K. G., \& Rice, J. B. 2003, A\&A, 399, 315

Strassmeier, K. G., Boyd, L. J., Epand, D. H., \& Granzer, T. 1997, PASP, 109, 697

Strassmeier, K. G., Pichler, T., Weber, M., \& Granzer, T. 2003, A\&A, 411, 595

Strassmeier, K. G., Rice, J. B., Ritter, A., et al. 2005, A\&A, 440, 1105

Wichmann, R., Schmitt, J. H. M. M., \& Hubrig, S. 2003, A\&A, 399, 983 
K. G. Strassmeier and J. B. Rice: Doppler imaging of PW And, Online Material $p 1$

\section{Online Material}




\section{Appendix A:}
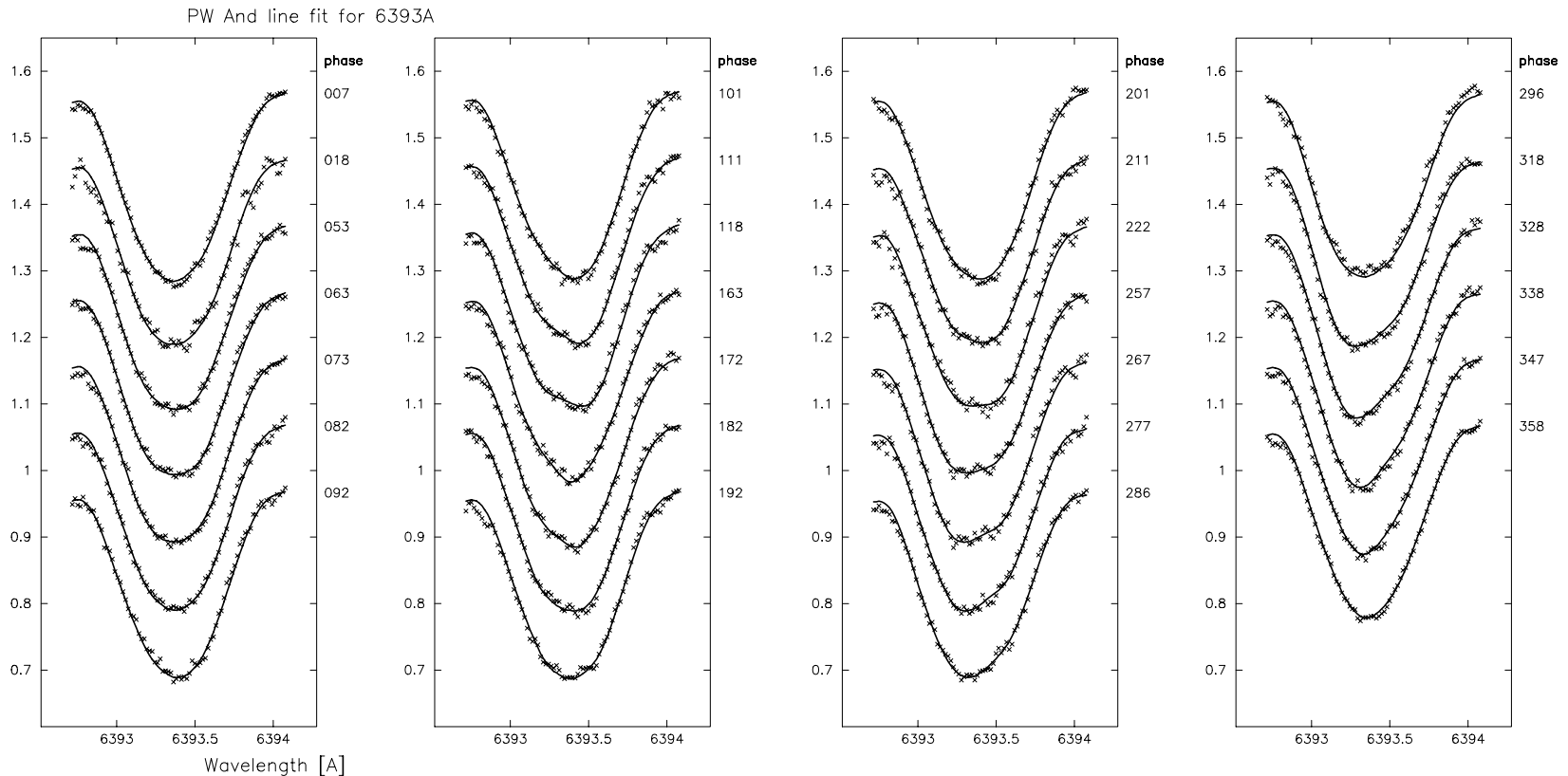

Fig. A.1. Fe I 639.3-nm line profile data and fits for all 27 phases. Phase is indicated in degrees $\left(0-360^{\circ}\right)$ on the right side of the individual graphs. Crosses are the data, the lines are the fits.
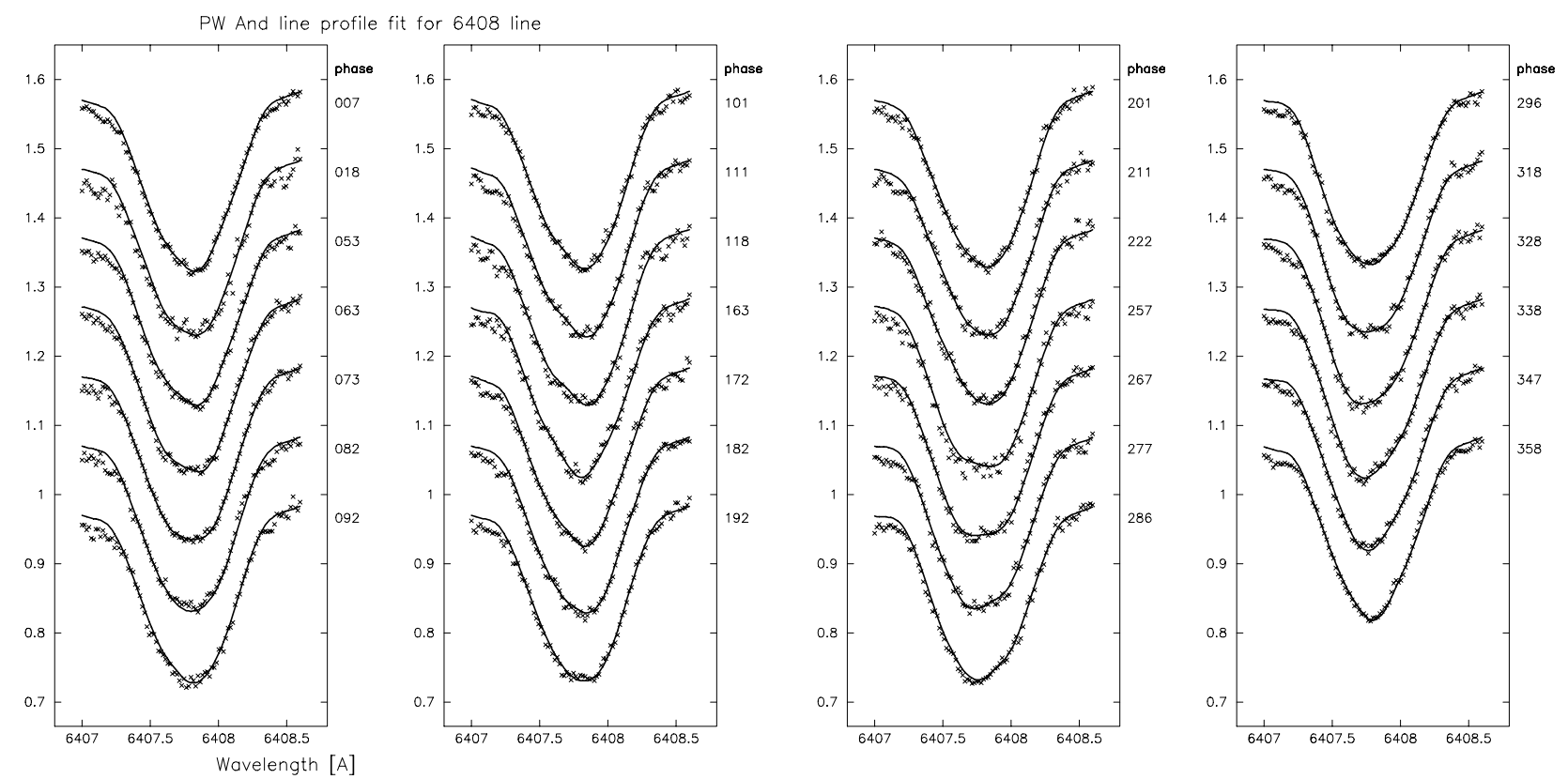

Fig. A.2. Fe I 640.8-nm line profile data and fits for all 27 phases. Phase is indicated in degrees $\left(0-360^{\circ}\right)$ on the right side of the individual graphs. Crosses are the data, the lines are the fits. 
K. G. Strassmeier and J. B. Rice: Doppler imaging of PW And, Online Material $p 3$
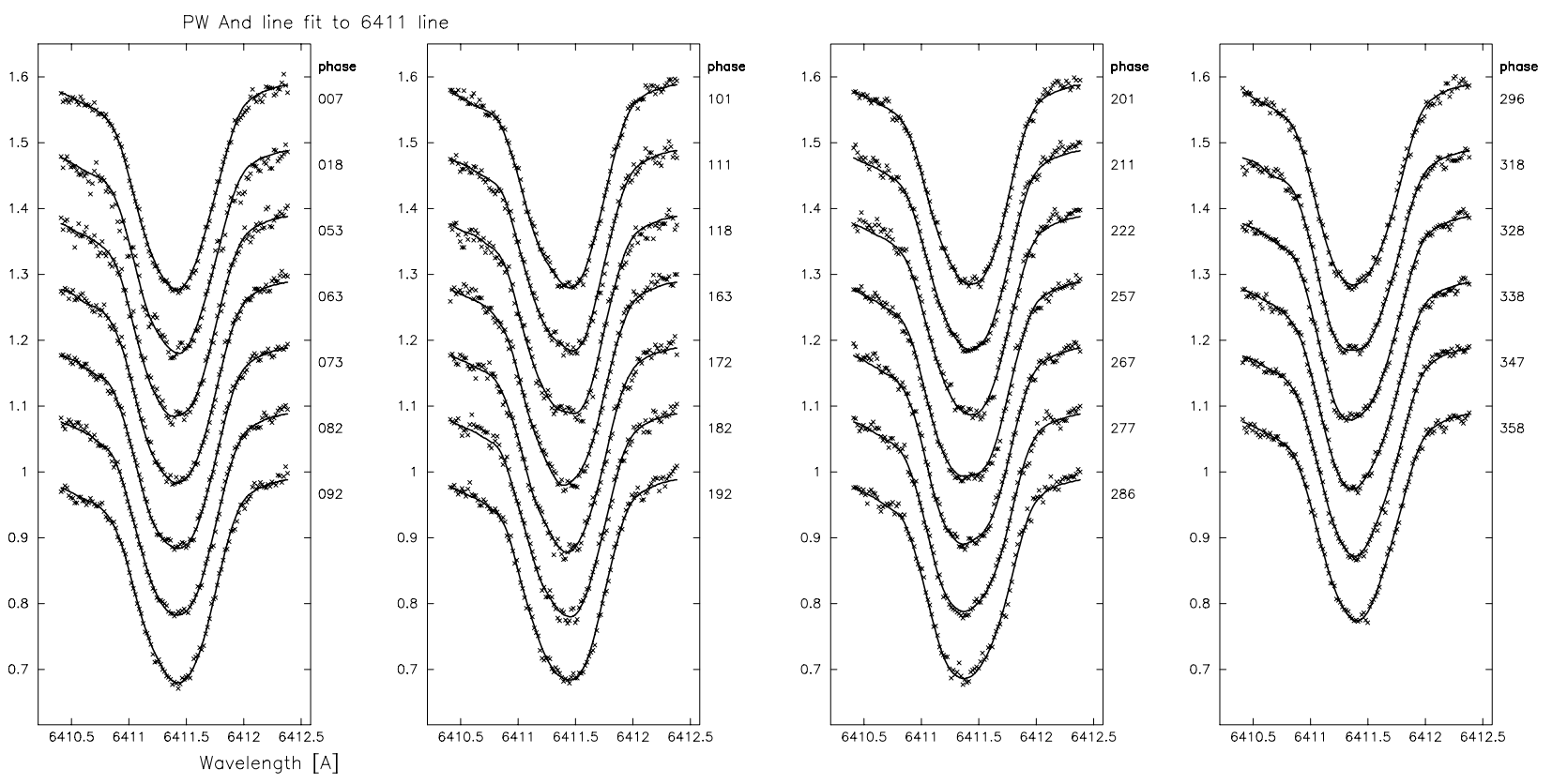

Fig. A.3. Fe I 641.1-nm line profile data and fits for all 27 phases. Phase is indicated in degrees $\left(0-360^{\circ}\right)$ on the right side of the individual graphs. Crosses are the data, the lines are the fits.
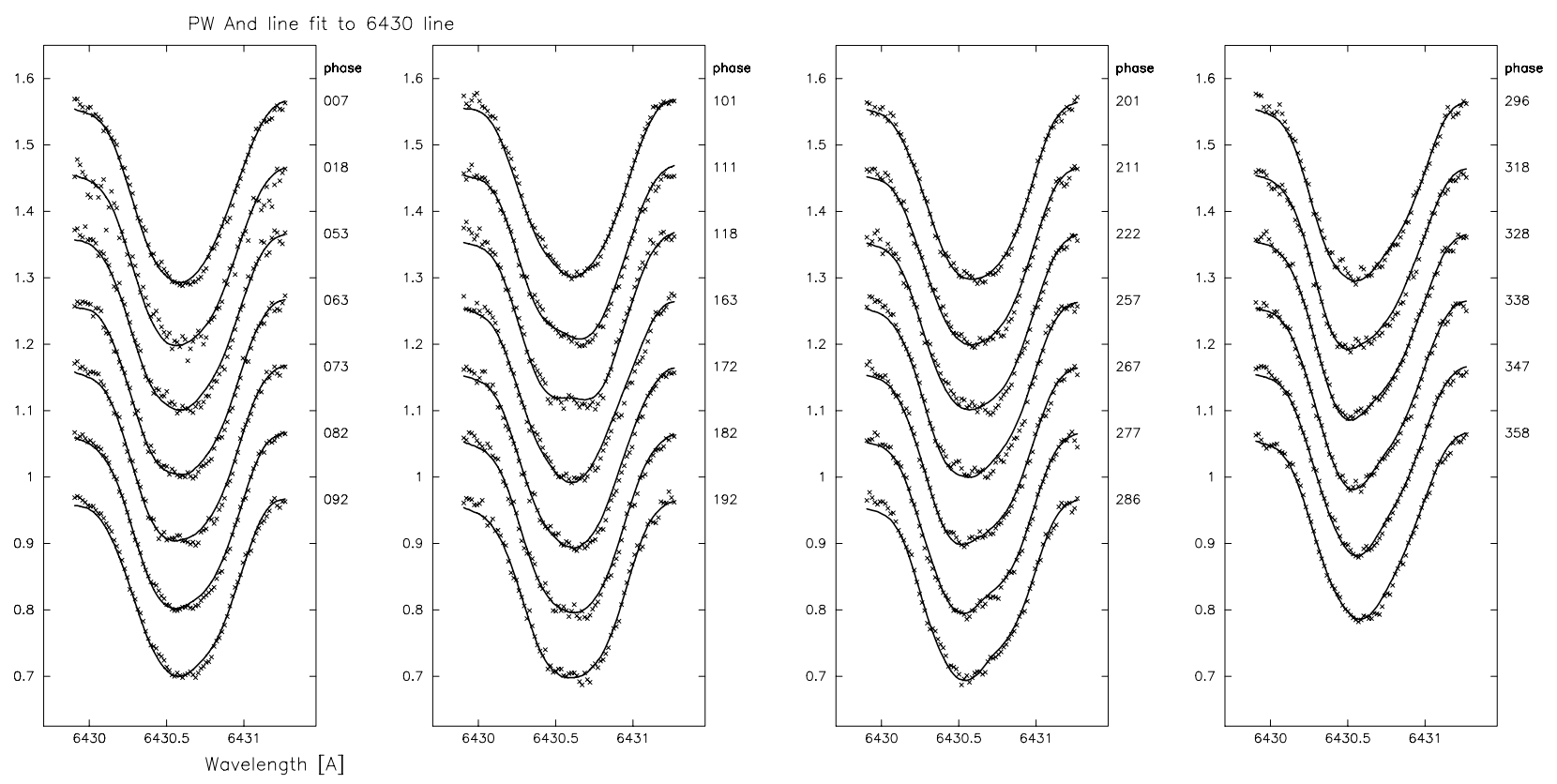

Fig. A.4. Fe I 643.0-nm line profile data and fits for all 27 phases. Phase is indicated in degrees $\left(0-360^{\circ}\right)$ on the right side of the individual graphs. Crosses are the data, the lines are the fits. 
K. G. Strassmeier and J. B. Rice: Doppler imaging of PW And, Online Material $p 4$
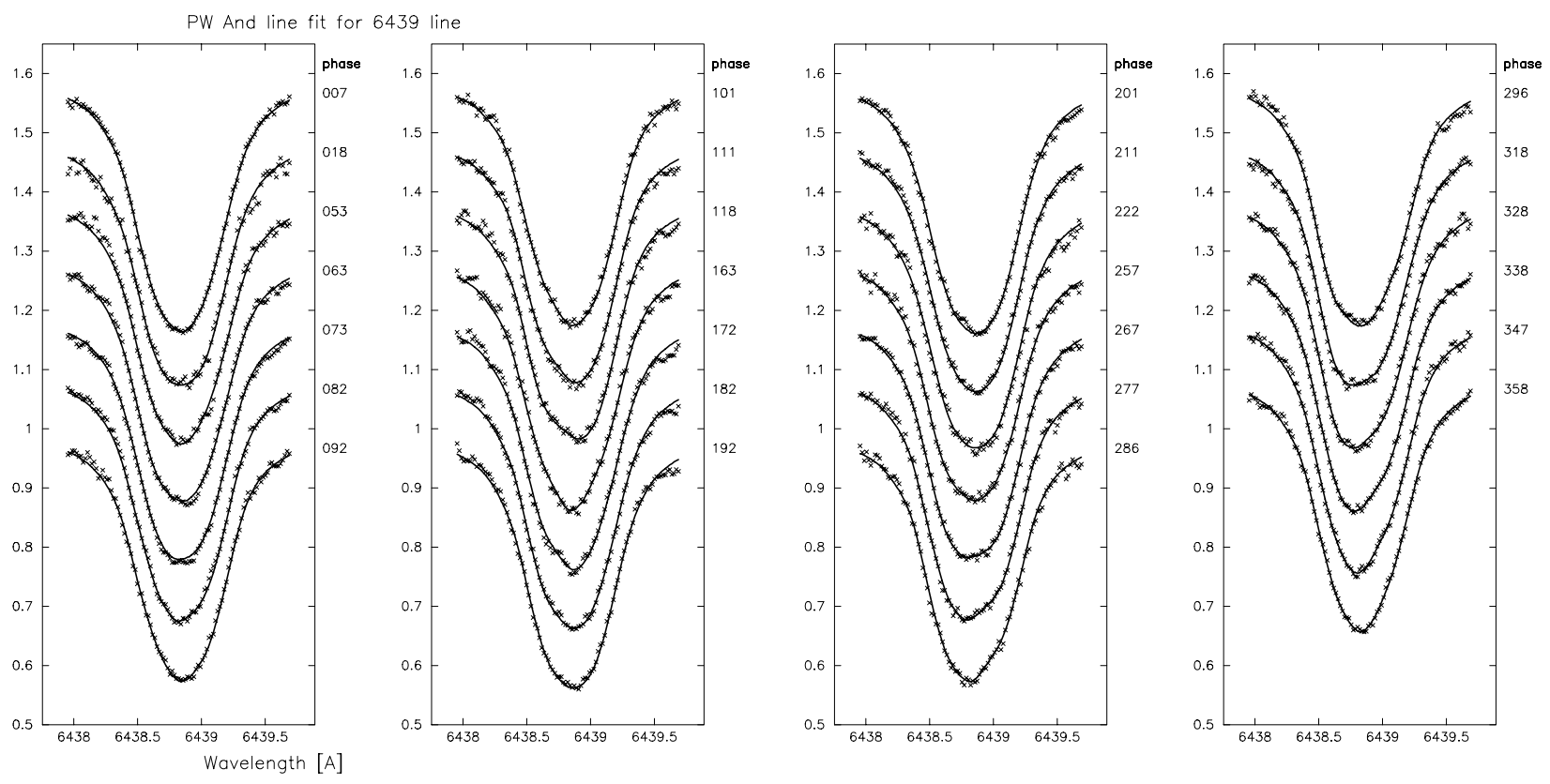

Fig. A.5. Ca I 643.9-nm line profile data and fits for all 27 phases. Phase is indicated in degrees $\left(0-360^{\circ}\right)$ on the right side of the individual graphs. Crosses are the data, the lines are the fits. 